\title{
Cohort study of depressed mood during pregnancy and after childbirth
}

\author{
Jonathan Evans, Jon Heron, Helen Francomb, Sarah Oke, Jean Golding, on behalf of the \\ Avon Longitudinal Study of Parents and Children Study Team
}

\begin{abstract}
Objective To follow mothers' mood through pregnancy and after childbirth and compare reported symptoms of depression at each stage.

Design Longitudinal cohort study.

Setting Avon.

Participants Pregnant women resident within Avon with an expected date of delivery between 1 April 1991 and 31 December 1992.

Main outcome measures Symptom scores from the Edinburgh postnatal depression scale at 18 and 32 weeks of pregnancy and 8 weeks and 8 months postpartum. Proportion of women above a threshold indicating probable depressive disorder.

Results Depression scores were higher at 32 weeks of pregnancy than 8 weeks postpartum (difference in means $0.88,95 \%$ confidence interval 0.79 to 0.97 ). There was no difference in the distribution of total scores or scores for individual items at the four time points. $1222(13.5 \%)$ women scored above threshold for probable depression at 32 weeks of pregnancy, $821(9.1 \%)$ at 8 weeks postpartum, and $147(1.6 \%)$ throughout. More mothers moved above the threshold for depression between 18 weeks and 32 weeks of pregnancy than between 32 weeks of pregnancy and 8 weeks postpartum.

Conclusions Symptoms of depression are not more common or severe after childbirth than during pregnancy. Research and clinical efforts need to be moved towards understanding, recognising, and treating antenatal depression.
\end{abstract}

\section{Introduction}

Women are more vulnerable to psychiatric illness during the postnatal period. The rate of psychiatric admission is increased postnatally, mostly because of the raised risk of psychosis in the first month after childbirth. ${ }^{1}$ In 1968, Pitt described a syndrome of "atypical depression following childbirth," although there is now no evidence that there is a categorical difference between depression after childbirth and depression at other times. The prevalence of nonpsychotic depressive illness in the postnatal period is similar to that in the general population. ${ }^{3}{ }^{4}$

Nevertheless, postnatal depression has become a focus of concern. General practitioners, health visitors, and others are exhorted to recognise and treat this condition. The consequences of postnatal depression to the child, mother, and family may include neglect of the child, family breakdown, self harm, and suicide. However, the more common consequences include emotional and behavioural problems, and cognitive delay in the children of depressed mothers. ${ }^{5}{ }^{6}$

In contrast, depression during pregnancy has been relatively neglected. Indeed, pregnancy was thought to protect women against depression. Studies of antenatal psychopathology have mostly examined antenatal mood as a predictor of postnatal depression. ${ }^{7-10}$ Watson et al found that in $23 \%$ of those who had postnatal depression this had started during pregnancy. ${ }^{11}$ Depressed mood during pregnancy has also been associated with poor attendance at antenatal clinics, substance misuse, low birth weight, and preterm delivery. ${ }^{12}{ }^{13}$ Psychopathological symptoms during pregnancy have physiological consequences for the fetus, which may explain some of these effects. ${ }^{14}$

We studied mood through pregnancy and after childbirth using prospectively gathered data from a cohort of 14000 women. We compared depressive symptom score, the pattern of reported symptoms, and the proportion of mothers above a threshold indicating probable depressive disorder at each stage.

\section{Participants and methods}

The Avon longitudinal study of parents and children enrolled women resident in Avon who were in the early stages of pregnancy with an expected date of delivery between 1 April 1991 and 31 December 1992. We recruited 14541 women, of whom 13799 had offspring surviving to 12 months old. Further details of the study aims and design are available (www.ich. bris.ac.uk/alspacext/). Ethical approval was obtained from the study's ethics committee and local ethics committees.

Women completed the Edinburgh postnatal depression scale ${ }^{15}$ and the Crown Crisp experiential index ${ }^{16}$ as part of a series of postal questionnaires. We present here data for questionnaires completed at 18 weeks and 32 weeks of pregnancy and at 8 weeks and 8 months postpartum. The Edinburgh postnatal depression scale focuses on the cognitive and affective features of depression rather than somatic symptoms. It is the only self report scale that has been validated for use postnatally and during pregnancy. ${ }^{17}{ }^{18}$ The scale cannot in itself confirm a diagnosis of depression; however, a score above 12 is widely used to indicate probable depressive disorder. Validation of the scale showed that all those found to have definite major depression when interviewed, had scored above 12 on the scale. Use of this threshold gave an overall sensitivity of $86 \%$ and specificity of $78 \%$ for all forms of depression. ${ }^{15}$

\section{Statistical methods}

We calculated mean Edinburgh postnatal depression scale scores for all responders and those responding at all four time points. Although the scores were negatively skewed, the differences in scores were symmetric and normal enough for analysis with paired $t$ tests. The $95 \%$ confidence intervals for the differences in the mean values were corrected for multiple (six) comparisons. We plotted frequency histograms of total scores for each period and compared changes in

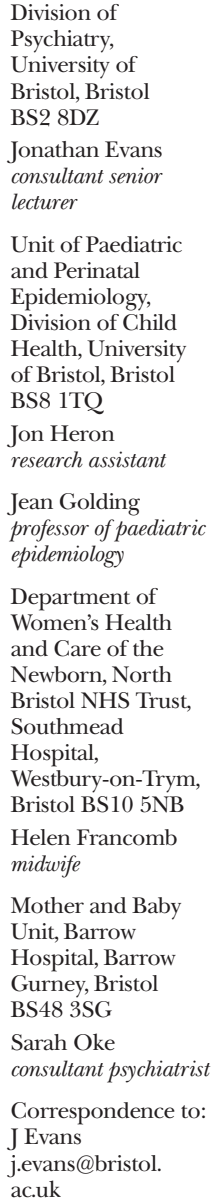

Unit of Paediatric and Perinatal

Epidemiology,

Division of Child

Health, University of Bristol, Bristol

BS8 1TQ

Jon Heron research assistant

Jean Golding professor of paediatric epidemiology

Department of Women's Health and Care of the Newborn, North Bristol NHS Trust, Southmead

Hospital,

Westbury-on-Trym, Bristol BS10 5NB

Helen Francomb

midwife

Mother and Baby Unit, Barrow

Hospital, Barrow Gurney, Bristol

BS48 3SG

Sarah Oke consultant psychiatrist

Correspondence to: J Evans

j.evans@bristol.

ac.uk

BMJ 2001;323:257-60 
Table 1 Edinburgh postnatal depression score during pregnancy and after childbirth

\begin{tabular}{lcccc} 
& $\begin{array}{c}\mathbf{1 8} \text { weeks } \\
\text { pregnant }\end{array}$ & $\begin{array}{c}\mathbf{3 2} \text { weeks } \\
\text { pregnant }\end{array}$ & $\begin{array}{c}\mathbf{8} \text { weeks } \\
\text { postpartum }\end{array}$ & $\begin{array}{c}\mathbf{8} \text { months } \\
\text { postpartum }\end{array}$ \\
\hline Women completing all four time points $(\mathrm{n}=9028):$ & & & \\
\hline Mean (SD) score & $6.62(4.66)$ & $6.72(4.94)$ & $5.84(4.65)$ & $5.25(4.61)$ \\
\hline No $(\%)$ depressed $($ score $\geqslant 13)$ & $1062(11.8)$ & $1222(13.6)$ & $831(9.2)$ & $731(8.1)$ \\
\hline Women completing any time points $(\mathrm{n}=12059):$ & & & \\
\hline No & 12059 & 11968 & 11579 & 11063 \\
\hline Mean (SD) score & $6.99(4.87)$ & $7.07(5.08)$ & $6.06(5.79)$ & $5.40(4.68)$ \\
\hline No $(\%)$ depressed $($ score $\geqslant 13)$ & $1676(13.9)$ & $1819(15.2)$ & $1181(10.2)$ & $973(8.8)$ \\
\hline
\end{tabular}

Table 2 Cumulative number (percentage) of women with various Edinburgh postnatal depression scores at each time point.

\begin{tabular}{lcccc} 
Score & $\begin{array}{c}\mathbf{1 8} \text { weeks } \\
\text { pregnant }\end{array}$ & $\begin{array}{c}\text { 32 weeks } \\
\text { pregnant }\end{array}$ & $\begin{array}{c}\mathbf{8} \text { weeks } \\
\text { postpartum }\end{array}$ & $\begin{array}{c}\mathbf{8} \text { months } \\
\text { postpartum }\end{array}$ \\
\hline 4 & $3412(37.8)$ & $3492(38.7)$ & $4163(46.1)$ & $4730(52.4)$ \\
\hline 12 & $7966(88.2)$ & $7806(86.5)$ & $8207(90.9)$ & $8290(91.8)$ \\
\hline 16 & $8742(96.8)$ & $8689(96.2)$ & $8766(97.1)$ & $8779(97.2)$ \\
\hline 20 & $8966(99.3)$ & $8951(99.1)$ & $8951(99.1)$ & $8972(99.4)$ \\
\hline 24 & $9022(99.9)$ & $9014(99.8)$ & $9010(99.8)$ & $9014(99.8)$ \\
\hline 28 & $9027(100)$ & $9028(100)$ & $9028(100)$ & $9021(99.9)$ \\
\hline
\end{tabular}

symptom score between 18 weeks and 32 weeks of pregnancy with changes between 32 weeks of pregnancy and 8 weeks postpartum using a paired $t$ test. Mean scores for each item of the Edinburgh postnatal depression scales at each time point were plotted to compare the frequency with which each symptom was reported. We repeated the analyses on the data from the Crown Crisp experiential index depression subscale to investigate whether a different scale produced any substantial differences in results.

We divided the cohort between probable cases of depression and probable non-cases at each time point using a threshold of 12/13 on the Edinburgh postnatal depression scale. We then compared changes across this threshold from 18 weeks to 32 weeks of pregnancy and 32 weeks of pregnancy to 8 weeks postpartum. We made a simplifying assumption that the two groups were independent, although some of the women would have been common to both groups. Under this assumption, using a normal approximation to the binomial distribution, we derived a confidence interval for the difference in the proportion of women rising above the threshold for probable depression between each time point.

\section{Results}

Of the 13799 eligible mothers, 12059 (87\%) completed at least one of the four questionnaires and $9028(65 \%)$ completed all four. Table 1 shows the mean Edinburgh postnatal depression scale scores for each period. Mean scores were higher in pregnancy than postnatally, with a peak at 32 weeks of pregnancy of 6.72 (SD 4.94) and a lowest value at 8 months postpartum (5.25 (4.61)). The mean change in depression score from that at 18 weeks of pregnancy was -0.097 (95\% confidence interval -0.18 to $-0.01, \mathrm{P}=0.025)$ at 32 weeks of pregnancy, $0.78(0.69$ to $0.88, \mathrm{P}<0.001)$ at 8 weeks postpartum, and 1.37 (1.27 to $1.46, \mathrm{P}<0.001)$ at 8 months postpartum. Mean change in score was 0.88 (0.79 to $0.97, \mathrm{P}<0.001)$ between 32 weeks of pregnancy and 8 weeks postpartum, 1.46 (1.37 to 1.56 , $\mathrm{P}<0.001)$ between 32 weeks of pregnancy and 8 months postpartum, and $-0.58(-0.50$ to -0.67 , $\mathrm{P}<0.001)$ between 8 weeks and 8 months postpartum. Women who did not complete all four questionnaires had higher depression symptom scores than women who completed all four. The mean depression scores were therefore higher when partial responders were included (table 1).

There was a small rise in depression symptom score during pregnancy (mean change 0.10 ; SE 0.043) and a small drop after childbirth $(-0.88 ; 0.047)$. These changes were significantly different (difference 0.98 , $95 \%$ confidence interval 0.83 to $1.13 ; \mathrm{P}<0.001$ ).

Table 2 shows the cumulative frequency of women with increasing Edinburgh postnatal depression scores at the four time points. The distribution did not differ between the time points.

Table 3 gives the scores for each item in the questionnaire. A similar pattern was seen at all four time points, but question 3 (I have blamed myself unnecessarily when things went wrong) was rated higher at 18 weeks of pregnancy that at other times and question 6 (things have been getting on top of me) was rated lower at 8 months postpartum than at other times.

When we repeated the above analyses using the Crown Crisp experiential index depression subscale there was no substantial differences in the findings.

The proportion of women with probable depression (Edinburgh postnatal depression score $\geqslant 13$ ) was $11.8 \%$ at 18 weeks of pregnancy, $13.5 \%$ at 32 weeks of pregnancy, $9.1 \%$ at 8 weeks postpartum, and $8.1 \%$ at 8 months postpartum (table 1). In all, 147 (1.6\%) women had probable depression at all four time points and $6771(75 \%)$ scored below the threshold at all time points; $436(4.8 \%)$ had probable depression at 32 weeks of pregnancy only and $240(2.7 \%)$ at 8 weeks postpartum only.

Of the 7966 women who were below the threshold for probable depression at 18 weeks of pregnancy, $673(8.4 \%)$ were above the threshold at 32 weeks of pregnancy. Of the 7806 below the threshold for probable depression at 32 weeks of pregnancy, 410 (5.3\%) were above the threshold at 8 weeks postpartum. There were $253(3.2 \%)$ fewer women newly rising above the threshold for probable depression after childbirth than during pregnancy $(95 \%$ confidence interval $2.4 \%$ to $4.0 \%$ ).

Table 3 Mean score for Edinburgh postnatal depression scale items at each time point among 9028 women with complete data. (Maximum score for each question is 3 )

\begin{tabular}{lcccc}
$\begin{array}{l}\text { Question } \\
\text { No }\end{array}$ & $\begin{array}{c}\mathbf{1 8} \text { weeks } \\
\text { pregnant }\end{array}$ & $\begin{array}{r}\text { 32 weeks } \\
\text { pregnant }\end{array}$ & $\begin{array}{c}\mathbf{8} \text { weeks } \\
\text { postpartum }\end{array}$ & $\begin{array}{c}\mathbf{8} \text { months } \\
\text { postpartum }\end{array}$ \\
\hline 1 & 0.33 & 0.41 & 0.31 & 0.29 \\
\hline 2 & 0.31 & 0.39 & 0.27 & 0.26 \\
\hline 3 & 1.20 & 1.02 & 1.01 & 1.01 \\
\hline 4 & 1.22 & 1.16 & 1.01 & 0.92 \\
\hline 5 & 0.59 & 0.69 & 0.55 & 0.49 \\
\hline 6 & 1.21 & 1.25 & 1.22 & 0.88 \\
\hline 8 & 0.32 & 0.39 & 0.23 & 0.25 \\
\hline 9 & 0.80 & 0.79 & 0.73 & 0.67 \\
\hline 10 & 0.49 & 0.54 & 0.44 & 0.40 \\
\hline & 0.14 & 0.08 & 0.07 & 0.08 \\
\hline
\end{tabular}




\section{Discussion}

We found that mothers have higher scores on the Edinburgh postnatal depression scale in pregnancy than postnatally and that the distribution of total scores and individual symptoms did not differ before and after childbirth. These data suggest that depression is no more likely after childbirth than it is after events during pregnancy.

\section{Validity of instrument}

Self report instruments do not provide a clinical diagnosis of depression. Some mothers scoring above the threshold will not have a depressive illness and some below the threshold will. However, a validation study found that a score of $\geqslant 13$ gave the best estimate of prevalence of depression. ${ }^{15}$ This threshold may overestimate depression during pregnancy. ${ }^{17}$ However, even if we used a higher threshold in pregnancy than postnatally, depression was still common during pregnancy. Furthermore, the proportion of women rating themselves as severely depressed was similar before and after childbirth. We found no evidence that the question asking whether the woman feared she might harm herself was misinterpreted or overrated during pregnancy, despite suggestions that this could happen. ${ }^{19}$

\section{Patterns of depression}

We found no evidence to support the existence of a subgroup of women with a specific type or severity of symptoms. It has been suggested that a small subgroup of women with postnatal depression have abnormal thyroid function leading to depressed mood. ${ }^{20}$ Women who did not respond at all time points had higher depression scores, and the non-responders probably included some of the most depressed women. Our results may therefore be biased by selective nonresponse among women with severe postnatal depression. Another explanation for our lower postnatal scores is that incidence of depression may peak at a time not measured in this study. Our data support research suggesting that there is no difference in the pattern of symptoms of depression during pregnancy or postnatally. Thus, postnatal depression does not seem to be a distinct syndrome.

The scales that we used were developed using only the core symptoms of depression because they are applicable to different contexts. Women's experience of depression during pregnancy and after childbirth, however, may be very different. Childbirth is an important biological, social, and psychological event.

Although caution is needed when equating self report data with a clinical diagnosis of depression, our data suggest that childbirth is less likely than the events of pregnancy to be followed by depression in women who are not depressed, and more likely to be followed by improvement in women who are depressed. The fall in probable cases of depression after childbirth has been reported in a study using this dataset and examining change and stability in depression using latent variable modelling. ${ }^{21}$ However, there is some evidence that childbirth may be a non-specific stressor for depression in most women and a specific stressor in a subgroup of women. ${ }^{22}$ We will be able to test this hypothesis by following mothers through to their next pregnancy.
What is known on this topic

Postnatal depression is common

Recognising and treating depression is emphasised in postnatal care

Depression is also common in pregnancy

What this study adds

Self reported symptom scores for depression are higher in pregnancy than postnatally

The severity and nature of depressed mood does not differ before and after childbirth

More mothers have scores that rise above a threshold for probable depression during pregnancy than after childbirth

\section{Implications}

The consequences of antenatal depression are not well understood. Psychopathology during pregnancy may have an important effect on the uterine environment, ${ }^{14}$ and research is urgently needed into the consequences for the child of antenatal depression.

It is also important to study the potential benefits of screening for, and treating, depression during pregnancy. Although there are concerns about the widespread use of antidepressants during pregnancy, the benefits may outweigh the risks for women with severe depression. Non-pharmacological interventions that have been found effective in mild to moderate depression ${ }^{23}$ could be evaluated for treating depression during pregnancy. ${ }^{24}$ Our results show depression during pregnancy is more common than postnatal depression. Offering treatment may be important for both the mother and the future wellbeing of the child and family.

We thank the mothers and fathers who took part and the midwives for their cooperation and help in recruitment. The study team comprises interviewers, computer technicians, laboratory technicians, clerical workers, research scientists, volunteers, and managers, who continue to make the study possible.

Contributors: JE wrote the paper and planned the data analyses, $\mathrm{JH}$ analysed the data, and $\mathrm{HF}$ planned the analyses and commented on the final draft of the paper. SO contributed to writing the paper and commented on the final draft. JG designed the study, advised on data analysis, and commented on the final draft. The ALSPAC study team designed the study and collected and entered the data. JE will act as guarantor.

Funding: Medical Research Council, Wellcome Trust, Department of Health, Department of the Environment, and various charitable organisations and commercial companies. The ALSPAC study is part of the WHO initiated European longitudinal study of pregnancy and childhood.

Competing interests: None declared.

1 Kendall RE, Wainwright S, Hailey A, Shannon B. The influence of childbirth on psychiatric morbidity. Psychol Med 1976;6:297-302.

2 Pitt B. Atypical depression following childbirth. $\mathrm{Br} J$ Psychiatry 1968; 114:1325-35.

3 Cooper PJ, Campbell EA, Day A, Kennerley H, Bond A. Non-psychotic psychiatric disorder after childbirth: a prospective study of prevalence, incidence, course and nature. Br J Psychiatry 1988;152:799-806.

4 O'Hara MW, Zekoski EM, Phillips LH, Wright EJ. Controlled prospective study of postpartum mood disorders: comparison of childbearing and non-childbearing women.J Abnormal Psychol 1990;99:3-15

5 Sinclair D, Murray L. Effects of postnatal depression on children's adjustment to school. Br J Psychiatry 1998;172:58-63.

6 Murray L, Sinclair D, Cooper P, Ducournau P, Turner P. The socioemotional development of 5 year olds with postnatally depressed mothers. J Child Psychol Psychiatry 1999;40:1259-71. 
7 O'Hara MW, Neunaber DJ, Zekoski EM. Prospective study of postpartum depression: prevalence, course and predictive factors. J Abnormal Psychol 1984:93:158-71

8 Green MJ. Who is unhappy after childbirth? Antenatal and intrapartum correlates from a prospective study. J Reprod Infant Psychol 1990:8:175-83.

9 Hobfoll SE, Ritter C, Lavin J, Hulsizer MR, Cameron RP. Depression prevalence and incidence among inner-city pregnant and postpartum prevalence and incidence among inner-city p
women. J Consult Clin Psychol 1995;65:445-53.

10 Cox JL, Connor Y, Kendell RE. Prospective study of the psychiatric disorders of childbirth. Br J Psychiatry 1982;140:111-7.

11 Watson JP, Elliott SA, Rugg AJ, Brough DI. Psychiatric disorder in pregnancy and the first postnatal year. Br J Psychiatry 1984;144:453-62.

12 Pagel MD, Smilkstein G, Regen H, Montano D. Psychosocial influences on new born outcome: a controlled prospective study. Soc Sci Med 1990;30:597-604.

13 Hedegaard M, Henriksen TB, Sabroe S, Secher NJ. Psychological distress in pregnancy and preterm delivery. BMJ 1993;307:234-9.

14 Teixeira JMA, Fisk NM, Glover V. Association between maternal anxiety in pregnancy and increased uterine artery resistance index: cohort based study. BMJ 1999;318:153-7.

15 Cox JL, Holden JM, Sagovsky R. Detection of postnatal depression. Development of the 10-item Edinburgh postnatal depression scale. $\mathrm{Br} \mathrm{J}$ Psychiatry 1987:150:782-6.

16 Crown S, Crisp AH. Manual of the Crown-Crisp experiential index. London: Hodder and Stoughton, 1979.
17 Murray D, Cox JL. Screening for depression during pregnancy with the Edinburgh depression scale (EPDS). J Reprod Infant Psychol 1990;8:99107

18 Thorpe K. A study of the Edinburgh postnatal depression scale for use with parent groups outside the postpartum period. J Reprod Infant Psychol 1993;11:119-25.

19 Green, JM, Snowdon C, Statham H. EPDS by post. Br J Psychiatry $1991 ; 158: 865$

20 Harris B, Othman S, Davies JA, Weppner GJ, Richards CJ, Newcombe RG, et al. Association between postpartum thyroid antibodies and depression. BMJ 1992;305:152-6.

21 Fergusson D, Horwood J, Thorpe K, ALSPAC Study Team. Changes in depression during and following pregnancy. Paediatric Perinatal Epidemiol $1996 ; 10 \cdot 279-93$.

22 Cooper PJ, Murray L. Course and recurrence of postnatal depression. evidence for the specificity of the diagnostic concept. Br J Psychiatry $1995 ; 166: 191-5$

23 Mynors-Wallis LM, Gath DH, Day A, Baker F. Randomised controlled trial of problem solving treatment, antidepressant medication, and combined treatment for major depression in primary care. BMJ 2000;320:26-3.

24 Spinelli MG. Interpersonal psychotherapy for depressed antepartum women: a pilot study. Am J Psychiatry 1997;154:1028-30.

(Accepted 19 April 2001)

\title{
Impact of published clinical outcomes data: case study in NHS hospital trusts
}

\author{
Russell Mannion, Maria Goddard
}

Centre for Health

Economics,

University of York, Heslington, York YO10 5DD

Russell Mannion senior research fellow Maria Goddard assistant director Correspondence to: R Mannion rm15@york.ac.uk

BMJ 2001;323:260-3

\begin{abstract}
Objective To examine the impact of the publication of clinical outcomes data on NHS Trusts in Scotland to inform the development of similar schemes elsewhere.

Design Case studies including semistructured interviews and a review of background statistics. Setting Eight Scottish NHS acute trusts. Participants 48 trust staff comprising chief executives, medical directors, stroke consultants, breast cancer consultants, nurse managers, and junior doctors.
\end{abstract} Main outcome measures Staff views on the benefits and drawbacks of clinical outcome indicators provided by the clinical resource and audit group (CRAG) and perceptions of the impact of these data on clinical practice and continuous improvement of quality.

Results The CRAG indicators had a low profile in the trusts and were rarely cited as informing internal quality improvement or used externally to identify best practice. The indicators were mainly used to support applications for further funding and service development. The poor effect was attributable to a lack of professional belief in the indicators, arising from perceived problems around quality of data and time lag between collection and presentation of data; limited dissemination; weak incentives to take action; a predilection for process rather than outcome indicators; and a belief that informal information is often more useful than quantitative data in the assessment of clinical performance.

Conclusions Those responsible for developing clinical indicator programmes should develop robust datasets. They should also encourage a working environment and incentives such that these data are used to improve continuously.

\section{Introduction}

The public dissemination of standardised data on clinical outcomes is now established practice in many health systems. In the United States, where public reporting is most advanced, comparative information on performance in the form of report cards, provider profiles, and consumer reports has been released for over a decade. ${ }^{1-3}$ In Europe, Scotland has been at the forefront of public disclosure. Since 1994 the Scottish Executive has published clinical outcome indicators collected by the clinical resource and audit group (CRAG) for all Scottish NHS acute trusts and health boards. More recently, clinical performance data have been published for trusts in England and Wales as part of the NHS performance assessment framework.

A postal questionnaire survey conducted by the Scottish Executive in 1997 indicated that the CRAG indicators published in Scotland were of some practical value to health professionals and, in a few instances, had helped to bring about a change in clinical practice. On the whole, however, the survey found that the indicators had little effect on behaviour. ${ }^{4} \mathrm{We}$ present the key findings of a study designed to examine the impact of the publication of these data on provider organisations. The CRAG indicators are similar to those now published more widely within the rest of the United Kingdom, and therefore an analysis of the Scottish experience may help with the implementation of such programmes elsewhere.

The CRAG indicators are compiled and disseminated by the Scottish Executive. Seven reports have been published detailing 38 clinical indicators for named trusts and health boards in Scotland. It is important to note that the CRAG indicators are not part of a formal framework of performance assessment. Since the indicators were first published the 